\title{
Characteristics and Factors Associated With the Mortality of Hypotensive Patients Attending the Emergency Department
}

\author{
Kumpol Amnuaypattanapon ${ }^{\mathrm{a}, \mathrm{b}}$, Suwimon Khansompop ${ }^{\mathrm{a}}$
}

\begin{abstract}
Background: The prevalence of hypotension in emergency departments (EDs) is approximately $1-2 \%$, but is associated with a mortality rate of $8-15 \%$. There has never been a study in Thailand examining the epidemiology or the risk factors for early mortality of patients presenting with hypotension in the ED. Therefore, this study aimed to define the characteristics, mortality rate within $48 \mathrm{~h}$ and associated factors of hypotensive patients at ED.
\end{abstract}

Methods: Data of patients with hypotension attending the ED of Thammasat University Hospital (TUH) were retrospectively studied.

Results: Of the 9,000 patients seen in the TUH ED, 233 were hypotensive for a prevalence of $2.5 \%$. Patients were old, with a mean age of $61 \pm 20$ years. The most common presenting symptom was fever, and sepsis was the most common cause of hypotension. The mean systolic blood pressure (SBP) was $78 \pm 8 \mathrm{~mm} \mathrm{Hg}$. Isotonic crystalloid volume resuscitation in first hour was $758 \mathrm{~mL}$ (interquartile range (IQR), $500-1,000)$ and the total volume to achieve a mean arterial pressure $(\mathrm{MAP}) \geq 65 \mathrm{~mm} \mathrm{Hg}$ was $1,142 \mathrm{~mL}$ (IQR, 500 - 1,500). Twenty-seven percent of patients needed vasopressor support. Nineteen patients died $\leq 48 \mathrm{~h}$, giving a case fatality rate of $8.2 \%$. Three independent factors associated with 48 -h mortality were initial pulse rate $>100$ beats/min (odds ratio (OR), $4.21 ; 95 \%$ confidence interval (CI), 1.05 - 16.88; $\mathrm{P}=0.042)$, diagnosis of shock (OR, 13.74 (1.49 - 126.61); $\mathrm{P}=0.021)$ and recurrent hypotension (OR, 6.91 (1.54 30.99); $\mathrm{P}=0.012$ ).

Conclusions: Hypotension in the ED was common and associated with high mortality rate. Better triage, patient monitoring and treatment may improve outcomes in these patients.

Keywords: Hypotension; Shock; Systolic blood pressure; Emergency department; Hypoperfusion

Manuscript submitted March 27, 2018, accepted April 9, 2018

aDepartment of Emergency Medicine, Faculty of Medicine, Thammasat University (Rangsit Campus), Pathum Thani 12121, Thailand

${ }^{\mathrm{b}}$ Corresponding Author: Kumpol Amnuaypattanapon, Department of Emergency Medicine, Faculty of Medicine, Thammasat University (Rangsit Campus), 99 Moo 18, Phahonyothin Road, Khlong Nueng, Khlong Luang, Pathum Thani 12121, Thailand. Email: kump29@gmail.com

doi: https://doi.org/10.14740/jocmr3422w

\section{Introduction}

Hypotension, defined as either a mean blood pressure (MBP) $<65 \mathrm{~mm} \mathrm{Hg}$, a systolic blood pressure (SBP) $<90 \mathrm{~mm} \mathrm{Hg}$ or a $>40 \mathrm{~mm} \mathrm{Hg}$ decrease in the SBP [1,2], is associated with a high mortality rate of $8-15 \%$ [3] in patients presenting to emergency departments (EDs). When hypotension progresses to shock or circulatory failure, there is an imbalance between tissue oxygen delivery and oxygen consumption that results in signs of poor perfusion, end-organ dysfunction and, if untreated, death [4].

In the USA, approximately 5.6 million hypotensive patients per year are seen in EDs, representing $1 \%$ of all ED attendances [5]. The main causes of hypotension are infection, hypovolemic, cardiogenic, neurogenic, and drug induced hypotension. However, the etiological characteristics and mortality rates in patients who develop shock in the ED are not well described.

The rapid diagnosis and treatment of hypotension is important because if shock develops, it may become irreversible and delayed treatment is associated with an increasing mortality of $35-56 \%$ for cardiogenic shock, and $40-60 \%$ for sepsisrelated shock [6]. Defining the clinical characteristics and epidemiology of hypotension are important to inform triage and management policies in EDs.

There are few data on the causes, clinical characteristic and prognosis of hypotension in the ED. We, therefore, set out to ascertain these and report the findings herein.

\section{Materials and Methods}

\section{Study design and patient population}

This was a retrospective cohort study of hypotensive patients presenting to the ED of Thammasat University Hospital (TUH) between June 1 and August 31,2016. TUH is a tertiary referral center situated in the northern outskirts of Bangkok with 700 beds and sees approximately 45,000 patient ED visits per year.

The study was approved by Human Research Ethics Committee of Thammasat University, and funding was obtained from the Thammasat Research Fund.

The study inclusion criteria were one of: 1) an $\mathrm{SBP}<90$ $\mathrm{mm} \mathrm{Hg}, 2$ ) a mean arterial blood pressure (MAP) $<65 \mathrm{~mm} \mathrm{Hg}$, 
or 3) a decrease in SBP $>40 \mathrm{~mm} \mathrm{Hg}$ from baseline $[5,6]$. The exclusion criteria were patient who aged $<17$ years old and/or who had an out of hospital cardiac arrest.

Using the hospital information system, all notes were retrieved of patients with a diagnosis of hypotension. Data were extracted using a standard case report form and included demographic data, major symptoms, serial pulse, blood pressure and oxygen saturation measurements, laboratory parameters, including serum lactate, clinical diagnoses and outcomes at 24 and $48 \mathrm{~h}$. The SIRS criteria were used to select patients, comprising at least two of the following criteria: HR $>90 / \mathrm{min}, \mathrm{RR}$ $>20 / \mathrm{min}$, body temperature $<36{ }^{\circ} \mathrm{C}$ or $>38.3{ }^{\circ} \mathrm{C}$, and white blood cell count $>12,000 / \mu \mathrm{L}$ or $<4,000 / \mu \mathrm{L}[2,7]$. We calculated the quick sequential organ failure assessment (qSOFA) score ranges from 0 to 3 with one point awarded for each of the following clinical signs: $\mathrm{SBP} \leq 100 \mathrm{~mm} \mathrm{Hg}, \mathrm{RR} \geq 22 / \mathrm{min}$, and altered mental status from baseline [7].

The sample size was estimated (STATA v14, Stata Corporation, USA) according to the previous report [8] and our pilot study, and the $48 \mathrm{~h}$ mortality rate of patients with hypotension was $8 \%$. Providing a power of $80 \%$ and two-side alpha of 0.05 , the calculated sample size was 223 .

\section{Statistical analysis}

Descriptive statistics are reported as frequencies, 95\% confidence intervals, mean (standard deviation (SD)) and median (interquartile range (IQR)). Proportional data between groups were analyzed by Chi-squared or Fisher's exact test; continuous data were analyzed by Student's $t$-test or the WilcoxonMann-Whitney $U$ test for normally distributed and skewed data, respectively. Factors associated with mortality within 48 $\mathrm{h}$ were analyzed by multivariable logistic regression. All analyses (STATA v14) were two sided and a statistically significant $\mathrm{P}$-value was defined as $<0.05$.

\section{Results}

A total of 9,000 patients were seen in the TUH ED during the 3 months study period. Of these, 250 patients presented with hypotension, including 17 patients who were referred to other hospitals. Therefore, the final number of included patients was 233. Patient's characteristics are shown in Table 1. The mean age was $61.4 \pm 20.2$ years, and there was a slightly higher proportion $(53.7 \%)$ of male patients.

The majority of patients had an underlying disease (69.1\%), including hypertension $(40.3 \%)$ and diabetes mellitus (DM, 26.2\%). Most patients $(88.8 \%$ ) had been brought to hospital by their families; the rest came by ambulance, and the emergency medical service (EMS, $11.2 \%$ ). A minority of patients, $2.2 \%$, were at the critical triage level, according to TUH triage criteria. The median times to receive initial IV fluids, to achieve an MAP $\geq 65 \mathrm{~mm} \mathrm{Hg}$, and admission times were 29 min, $103 \mathrm{~min}$, and $21 \mathrm{~h}$, respectively.

The chief complaints were fever (18.5\%), dyspnea $(16.7 \%)$, and abdominal pain (8.6\%). On admission to the ED, the mean SBP was $78 \mathrm{~mm} \mathrm{Hg}$, the mean diastolic (DBP) was $48 \mathrm{~mm} \mathrm{Hg}$, the mean pulse rate was $96 / \mathrm{min}$, and $7.3 \%$ of patients had an oxygen saturation $\leq 90 \%$. The mean shock index (heart rate/SBP) and modified shock index (heart rate/MAP) were 1.3 and 1.7 , respectively. Of patients, $36.5 \%$ had $\geq 2$ of the SIRS criteria and $21.9 \%$ met qSOFA score $\geq 2$. The most common cause of hypotension was infection (50.2\%) followed by hypovolemia $(40.8 \%)$. The initial median serum lactate concentration was $3.5 \mathrm{mmol} / \mathrm{L}$ (IQR 1.6 - 4.4).

The median volume of fluid required to achieve an MAP $\geq 65 \mathrm{~mm} \mathrm{Hg}$ was 1,142 mL (IQR $500-1,500$ ) and $27.0 \%$ of patients received a vasopressor. Of patients, $40.3 \%$ had recurrent hypotension, $16.7 \%$ of patients were intubated, and $8.2 \%$ died within $48 \mathrm{~h}$.

By univariable analysis, the factors associated with mortality within $48 \mathrm{~h}$ are shown in Table 2 . The main factors were: 1) being bed ridden, 2) initial pulse rate $>100$ beats $/ \mathrm{min}, 3$ ) initial pulse oximetry $\leq 90 \%, 4$ ) altered mental status, 5) qSOFA score $\geq 2,6$ ) diagnosis of shock, 7) total fluid in first hour $\geq 1,500 \mathrm{~mL}, 8$ ) intubation, 9) recurrent hypotension, and 10) those who needed a vasopressor. By multivariable logistic regression, there were three independent factors associated with death $\leq 48 \mathrm{~h}$ (Table 3 ); these were initial pulse rate $>100$ beats/ min, diagnosis of shock, and recurrent hypotension.

\section{Discussion}

In our retrospective study, we have shown that hypotensive patients account for less than 3\% of all ED attendances, but they suffered a 48 -h mortality of just over $8 \%$ and half of those deaths were associated with a final diagnosis of infection.

The baseline characteristics of our ED patients presenting with hypotension were similar to those reported in previous studies, i.e. older patients with underlying morbidities [8-10]. Waiting times to see the doctor and receive treatment were 10 and $28 \mathrm{~min}$, respectively, which compare favorably to the times reported by the US CDC in 2010 - 2011 of 10 and $30 \mathrm{~min}$, respectively. Our ED is busy and sees some 45,000 patients per year and overcrowding and long waiting times are significant challenges we face.

The prevalence of hypotension in EDs varies depending on where the study took place and the definition used of hypotension. Moreover, definitions of sepsis and septic shock have evolved over time with changing definitions of hypotension and shock. Holler et al from Denmark reported an ED prevalence of hypotension of $1 \%$ with a 7-day mortality of $12 \%$ [1]; this contrasts with a higher rate of $19 \%$ reported by Jones et al whose patients suffered an in-hospital mortality of $8 \%$ [8].

Although the most common complaint was fever, it was only reported in a little under $20 \%$ of patients; interestingly a similar proportion also had a measured fever of $\geq 38^{\circ} \mathrm{C}$. This should alert physicians to a diagnosis of possible sepsis even if the measured temperature is within the normal range. In our series, 113 of 233 patients had sepsis according to qSOFA score, of which $45 \%$ had septic shock. This score has been accepted as a screening tool to identify patients who may have early sepsis in the EDs $[7,11]$. 
Table 1. Baseline Characteristics and Clinical Outcomes of 233 Hypotensive Patients

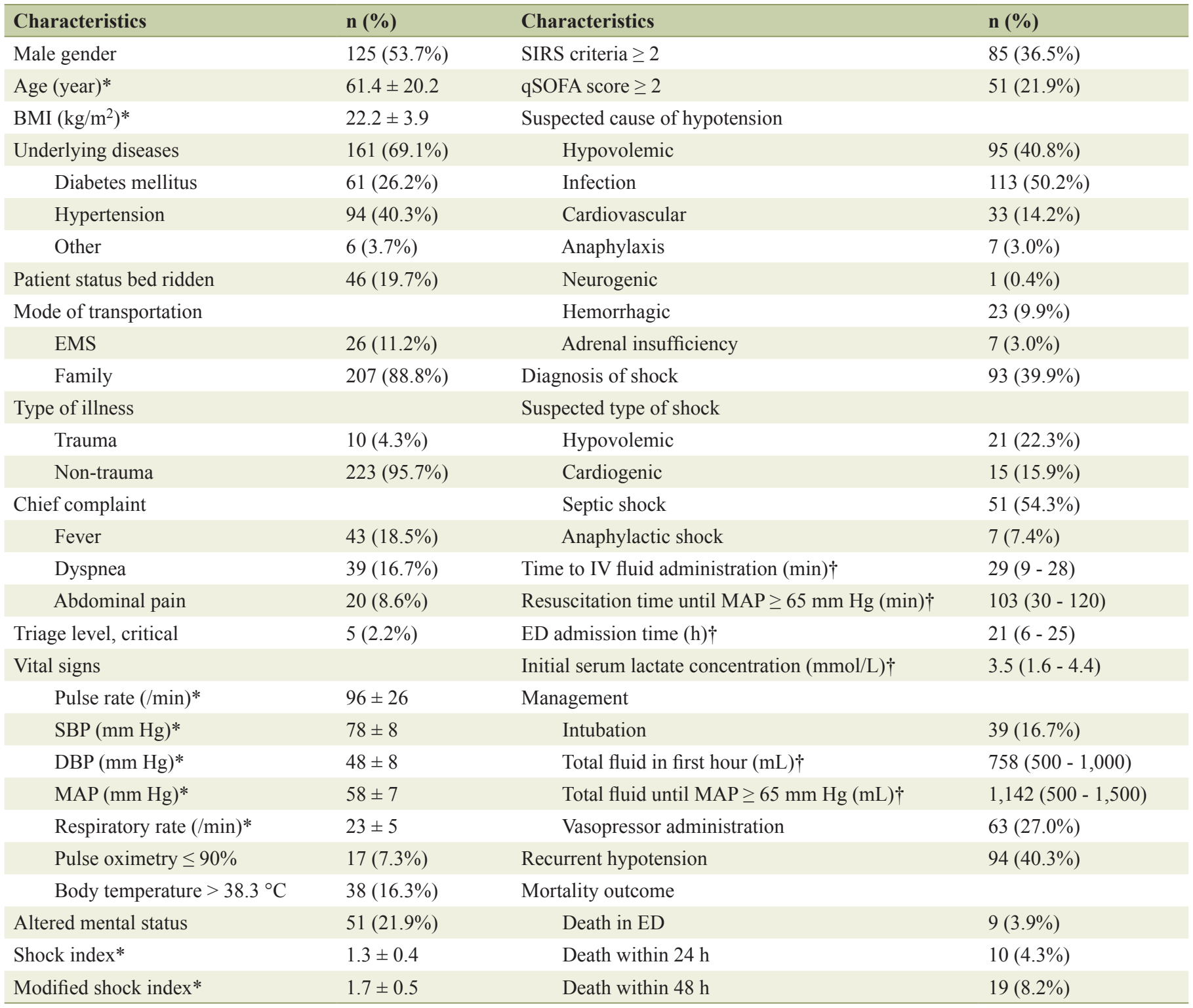

${ }^{*}$ Data are expressed as mean \pm SD. †Data are expressed as median (IQR). BMI: body mass index; EMS: emergency medical service; ED: emergency department; SBP: systolic blood pressure; DBP: diastolic blood pressure; MAP: mean arterial pressure. Shock index = heart rate/systolic blood pressure. Modified shock index = heart rate/MAP. SIRs: systemic inflammatory responses; qSOFA score: quick sequential organ failure assessment score; IV: intravenous.

We found that the qSOFA score was related to hospital mortality in the univariable analysis but it was not a significant factor in the multivariable analysis, which accords with Askim et al and Tusgul et al who found that the qSOFA failed to be a risk stratification tool for predicting 7- and 30-day mortality $[12,13]$. The shock and modified shock index may be good markers of a patient's hemodynamic status and have been found to be associated with increased mortality in the ED $[14,15]$. We did not show that either index was a significant mortality factor. This difference could be explained by heterogeneity in patients' characteristics and clinical settings among studies.
Within the first hour, the mean volume of isotonic crystalloid fluid resuscitation given to our patients was approximately $750 \mathrm{~mL}$. This amount is in accordance to the survival sepsis campaign, which recommends fluid replacement of $30 \mathrm{~mL} / \mathrm{kg}$ in the first $3 \mathrm{~h} \mathrm{[16].}$

The three independent factors associated with death within $48 \mathrm{~h}$ were initial pulse rate $>100$ beats/min, shock diagnosis, and recurrent hypotension; these are consistent with the findings of Barfod et al who reported that the $\mathrm{SpO}_{2}$, respiratory rate, SBP and Glasgow coma score were all significantly associated with in-hospital mortality [17]. Factors that we found to be unrelated to increased mortality include serum lactate level 
Table 2. Univariable Analysis of Factors Associated With $48 \mathrm{~h}$ Mortality in Hypotensive Patients

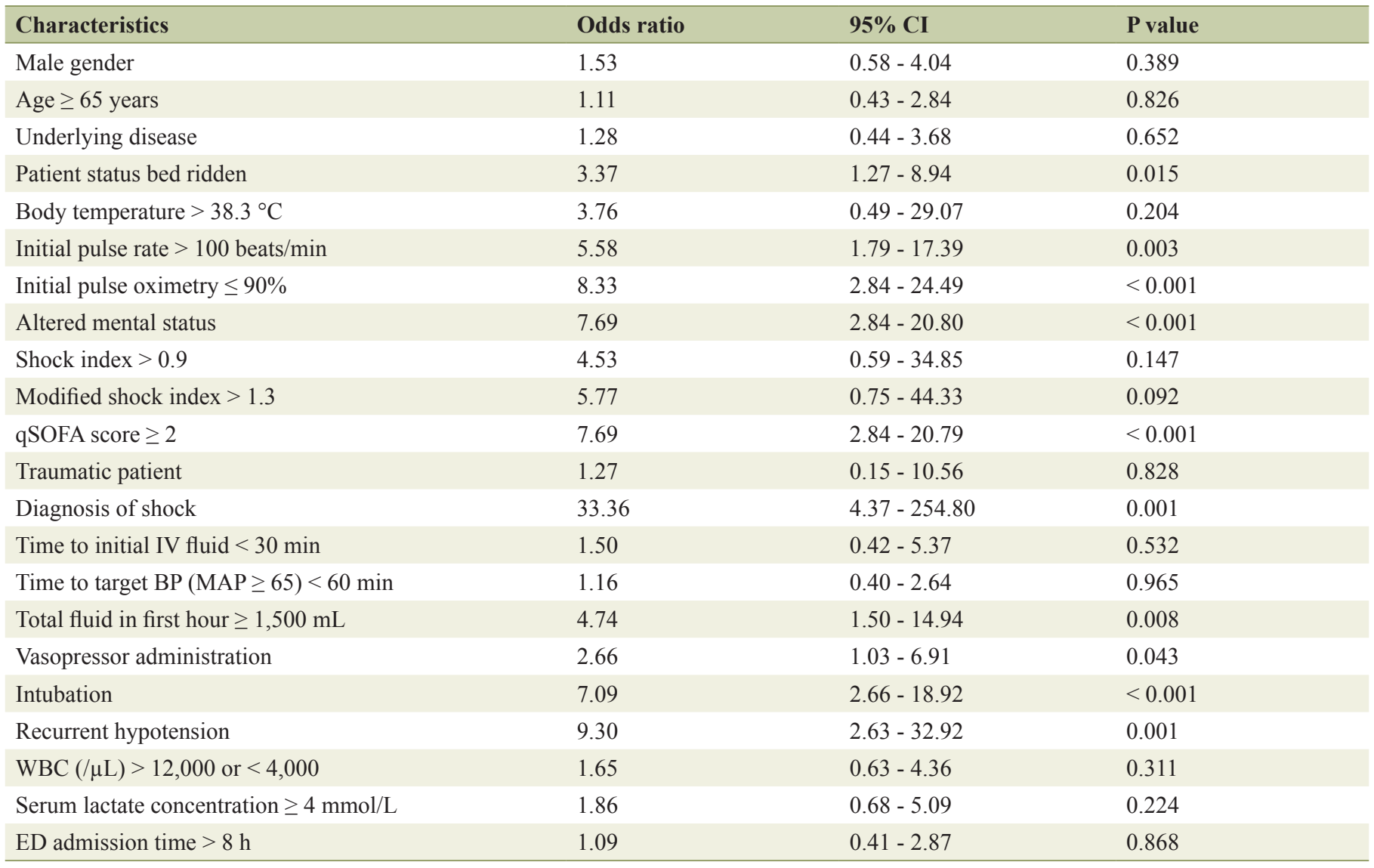

EMS: emergency medical service; MAP: mean arterial pressure; Cl: confidence interval; ED: emergency department; SBP: systolic blood pressure; IV: intravenous. Shock index = heart rate/systolic blood pressure. Modified shock index = heart rate/MAP. qSOFA score: quick sequential organ failure assessment score; WBC: white blood cell count.

and shock index which contrast to a number of other studies [14, 18-22], and could be due partly to our heterogeneous population. Interestingly, a recent meta-analysis found that delayed antibiotic treatment was not associated with an increased risk of death in sepsis [22].
Our study had some limitations. This was a single hospital study with a relatively small number of patients. In addition, this was retrospective, with some incompleteness of data. Larger prospective studies are needed to validate our findings, which could contribute to developing a predictive score of

Table 3. Multivariable Analysis of Factors Associated With 48 h Mortality in Hypotensive Patients

\begin{tabular}{llll}
\hline Characteristics & Odds ratio & $\mathbf{9 5 \%}$ CI & P value \\
\hline Patient status bed ridden & 2.24 & $0.63-8.00$ & 0.214 \\
Initial pulse rate $>100$ beats/min & 4.21 & $1.05-16.88$ & 0.042 \\
Initial pulse oximetry $\leq 90 \%$ & 2.94 & $0.68-12.74$ & 0.149 \\
qSOFA score $\geq 2$ & 3.13 & $0.94-10.30$ & 0.061 \\
Altered mental status & 2.20 & $0.60-8.04$ & 0.235 \\
Diagnosis of shock & 13.74 & $1.49-126.61$ & 0.021 \\
Intubation & 1.88 & $0.52-6.82$ & 0.337 \\
Total fluid in first hour $\geq 1,500 \mathrm{~mL}$ & 3.79 & $0.72-20.00$ & 0.117 \\
Vasopressor administration & 0.53 & $0.15-1.86$ & 0.322 \\
Recurrent hypotension & 6.91 & $1.54-30.99$ & 0.012 \\
\hline
\end{tabular}

$\mathrm{Cl}$ : confidence interval; qSOFA score: quick sequential organ failure assessment score. 
mortality among these patients.

\section{Conclusions}

Hypotension is a common problem in the ED, with sepsis being the most common cause. Our 48-h mortality rate of all causes hypotension was high. In our setting, initial pulse rate $>$ 100 beats/min, diagnosis of shock, and recurrent hypotension were risk factors for death. This knowledge should help ED personals to better triage these patients, and initiate treatment promptly.

\section{Acknowledgments}

We thank Dr. Bob Taylor, Dr. Sith Siramolpiwat and Dr. Sumet Kongkiatpaiboon for proof-reading and reviewing the manuscript.

\section{Funding Support}

The research was supported by Thammasat Research Fund.

\section{Conflict of Interest}

None.

\section{References}

1. Vincent JL, De Backer D. Circulatory shock. N Engl J Med. 2013;369(18):1726-1734.

2. Dellinger RP, Levy MM, Rhodes A, Annane D, Gerlach H, Opal SM, Sevransky JE, et al. Surviving Sepsis Campaign: international guidelines for management of severe sepsis and septic shock, 2012. Intensive Care Med. 2013;39(2):165-228.

3. Holler JG, Bech CN, Henriksen DP, Mikkelsen S, Pedersen C, Lassen AT. Nontraumatic hypotension and shock in the emergency department and the prehospital setting, prevalence, etiology, and mortality: a systematic review. PLoS One. 2015;10(3):e0119331.

4. Kumar A, Parillo JE. Shock. Classification, pathophysiology, and approach to management in critical care medicine: principles of diagnosis and management in the adult, 3rd ed. Philadelphia, PA: Mosby Inc., 2008:379. Book Chapters.

5. Pitts SR, Niska RW, Xu J, Burt CW. National hospital ambulatory medical care survey: 2006 emergency department summary. Natl Health Stat Report. 2008;7:1-38.

6. Bai X, Yu W, Ji W, Lin Z, Tan S, Duan K, Dong Y, et al. Early versus delayed administration of norepinephrine in patients with septic shock. Crit Care. 2014;18(5):532.

7. Seymour CW, Liu VX, Iwashyna TJ, Brunkhorst FM, Rea TD, Scherag A, Rubenfeld G, et al. Assessment of clinical criteria for sepsis: for the third international consensus definitions for sepsis and septic shock (Sepsis-3). JAMA. 2016;315(8):762-774.

8. Jones AE, Yiannibas V, Johnson C, Kline JA. Emergency department hypotension predicts sudden unexpected in-hospital mortality: a prospective cohort study. Chest. 2006;130(4):941-946.

9. Quach JL, Downey AW, Haase M, Haase-Fielitz A, Jones D, Bellomo R. Characteristics and outcomes of patients receiving a medical emergency team review for respiratory distress or hypotension. J Crit Care. 2008;23(3):325331.

10. Jones AE, Stiell IG, Nesbitt LP, Spaite DW, Hasan N, Watts BA, Kline JA. Nontraumatic out-of-hospital hypotension predicts inhospital mortality. Ann Emerg Med. 2004;43(1):106-113.

11. Singer AJ, Ng J, Thode HC, Jr., Spiegel R, Weingart S. Quick SOFA scores predict mortality in adult emergency department patients with and without suspected infection. Ann Emerg Med. 2017;69(4):475-479.

12. Askim A, Moser F, Gustad LT, Stene H, Gundersen M, Asvold BO, Dale J, et al. Poor performance of quickSOFA (qSOFA) score in predicting severe sepsis and mortality - a prospective study of patients admitted with infection to the emergency department. Scand J Trauma Resusc Emerg Med. 2017;25(1):56.

13. Tusgul S, Carron PN, Yersin B, Calandra T, Dami F. Low sensitivity of qSOFA, SIRS criteria and sepsis definition to identify infected patients at risk of complication in the prehospital setting and at the emergency department triage. Scand J Trauma Resusc Emerg Med. 2017;25(1):108.

14. Liu YC, Liu JH, Fang ZA, Shan GL, Xu J, Qi ZW, Zhu $\mathrm{HD}$, et al. Modified shock index and mortality rate of emergency patients. World J Emerg Med. 2012;3(2):114-117.

15. Rady MY, Smithline HA, Blake H, Nowak R, Rivers E. A comparison of the shock index and conventional vital signs to identify acute, critical illness in the emergency department. Ann Emerg Med. 1994;24(4):685-690.

16. Howell MD, Davis AM. Management of sepsis and septic shock. JAMA. 2017;317(8):847-848.

17. Barfod C, Lauritzen MM, Danker JK, Soletormos G, Forberg JL, Berlac PA, Lippert F, et al. Abnormal vital signs are strong predictors for intensive care unit admission and in-hospital mortality in adults triaged in the emergency department - a prospective cohort study. Scand J Trauma Resusc Emerg Med. 2012;20:28.

18. Shapiro NI, Howell MD, Talmor D, Nathanson LA, Lisbon A, Wolfe RE, Weiss JW. Serum lactate as a predictor of mortality in emergency department patients with infection. Ann Emerg Med. 2005;45(5):524-528.

19. Sloan EP, Koenigsberg M, Clark JM, Weir WB, Philbin $\mathrm{N}$. Shock index and prediction of traumatic hemorrhagic shock 28-day mortality: data from the DCLHb resuscitation clinical trials. West J Emerg Med. 2014;15(7):795802.

20. Sterling SA, Miller WR, Pryor J, Puskarich MA, Jones AE. The impact of timing of antibiotics on outcomes in severe sepsis and septic shock: a systematic review and meta-analysis. Crit Care Med. 2015;43(9):1907-1915. 
21. Berger T, Green J, Horeczko T, Hagar Y, Garg N, Suarez A, Panacek E, et al. Shock index and early recognition of sepsis in the emergency department: pilot study. West J Emerg Med. 2013;14(2):168-174.
22. Cevik AA, Dolgun H, Oner S, Tokar B, Acar N, Ozakin E, Kaya F. Elevated lactate level and shock index in nontraumatic hypotensive patients presenting to the emergency department. Eur J Emerg Med. 2015;22(1):23-28. 